\title{
Comparison of Poisson structures on moduli spaces
}

\author{
Indranil Biswas ${ }^{1}$. Francesco Bottacin ${ }^{2} \cdot$ Tomás L. Gómez ${ }^{3}$ (I)
}

Received: 19 April 2021 / Accepted: 3 December 2021 / Published online: 28 December 2021

(c) The Author(s) 2021

\begin{abstract}
Let $X$ be a complex irreducible smooth projective curve, and let $\mathbb{L}$ be an algebraic line bundle on $X$ with a nonzero section $\sigma_{0}$. Let $\mathcal{M}$ denote the moduli space of stable Hitchin pairs $(E, \theta)$, where $E$ is an algebraic vector bundle on $X$ of fixed rank $r$ and degree $\delta$, and $\theta \in H^{0}\left(X, \mathcal{E} n d(E) \otimes K_{X} \otimes \mathbb{L}\right)$. Associating to every stable Hitchin pair its spectral data, an isomorphism of $\mathcal{M}$ with a moduli space $\mathcal{P}$ of stable sheaves of pure dimension one on the total space of $K_{X} \otimes \mathbb{L}$ is obtained. Both the moduli spaces $\mathcal{P}$ and $\mathcal{M}$ are equipped with algebraic Poisson structures, which are constructed using $\sigma_{0}$. Here we prove that the above isomorphism between $\mathcal{P}$ and $\mathcal{M}$ preserves the Poisson structures.
\end{abstract}

Keywords Spectral data - Hitchin pair · Poisson structure · Hypercohomology

Mathematics Subject Classification 53D17 · 53D30 $\cdot 14 \mathrm{H} 60 \cdot 14 \mathrm{~J} 60$

\section{Introduction}

Let $X$ be a complex irreducible smooth projective curve of genus $g$. Take an algebraic line bundle $N$ on $X$ such that $N \otimes K_{X}^{-1}$ admits a nonzero section, where $K_{X}$ is the

Tomás L. Gómez

tomas.gomez@icmat.es

Indranil Biswas

indranil@math.tifr.res.in

Francesco Bottacin

bottacin@math.unipd.it

1 School of Mathematics, Tata Institute of Fundamental Research, Homi Bhabha Road, Mumbai 400005, India

2 Dipartimento di Matematica, Università degli Studi di Padova, Via Trieste 63, 35121 Padova, Italy

3 Instituto de Ciencias Matemáticas (CSIC-UAM-UC3M-UCM), Nicolás Cabrera 15, Madrid, Campus Cantoblanco UAM, 28049, Spain 
canonical bundle of $X$; fix a section $\sigma_{0} \in H^{0}\left(X, N \otimes K_{X}^{-1}\right) \backslash\{0\}$. Fix integers $r$ and $\delta$. Let $\mathcal{M}$ denote the moduli space of stable pairs of the form $(E, \theta)$, where $E$ is an algebraic vector bundle on $X$ of rank $r$ and degree $\delta$, and $\theta \in H^{0}(X, \mathcal{E} n d(E) \otimes N)$. These are called Hitchin pairs; when $N=K_{X}$, they are called Higgs bundles. The moduli space $\mathcal{M}$ is nonempty if one of the following four assumptions hold (cf. [13, Remark 3.4]):

(1) the genus $g$ of $X$ is at least 2;

(2) $g=1, N=K_{X}$ and $\operatorname{gcd}(r, \delta)=1$;

(3) $g=1$ and $\operatorname{deg}\left(N \otimes K_{X}^{-1}\right)>0$; and

(4) $g=0$ and $\operatorname{deg}\left(N \otimes K_{X}^{-1}\right) \geq 3$.

We assume that one of these four conditions hold. This moduli space $\mathcal{M}$ has a natural algebraic Poisson structure $[4,13]$, which is constructed using $\sigma_{0}$.

In the special case where $N=K_{X}$ and $\sigma_{0}$ is the constant function 1 , this $\mathcal{M}$ is a moduli space of Higgs bundles. In that case, the Poisson structure is nondegenerate, meaning it is a symplectic structure; this symplectic structure was constructed earlier in $[8,9]$. Furthermore, there is a natural algebraic 1 -form on $\mathcal{M}$ such that the symplectic form is the exterior derivative of it.

Let $S$ denote the smooth complex quasi-projective surface defined by the total space of the line bundle $N$. Given any Hitchin pair $(E, \theta) \in \mathcal{M}$, there is a subscheme $Y_{(E, \theta)} \subset S$ of dimension one associated to it. Furthermore, associated to the pair $(E, \theta)$ there is a coherent sheaf $\mathcal{L}_{(E, \theta)}$ on $Y_{(E, \theta)}$ which is pure of dimension one. This pair $\left(Y_{(E, \theta)}, \mathcal{L}_{(E, \theta)}\right)$ is known as the spectral datum associated to $(E, \theta)$. This construction produces an algebraic map

$$
\Phi: \mathcal{M} \longrightarrow \mathcal{P}
$$

where $\mathcal{P}$ is a moduli space of stable sheaves of pure dimension one on $S$. This construction is reversible, and $\Phi$ is in fact an isomorphism (see $[1,8,9,17]$ ).

The earlier mentioned section $\sigma_{0}$ of $N \otimes K_{X}^{-1}$ produces a Poisson structure on the surface $S$. An algebraic Poisson structure on $\mathcal{P}$ is constructed using this Poisson structure on $S[5,18]$.

It may be mentioned that when $N=K_{X}$ and $\sigma_{0}$ is the constant function 1 , the Poisson structure on $S$ is the canonical symplectic form on the cotangent bundle $K_{X}$ of $X$. In that case the Poisson structure on $\mathcal{P}$ coincides with the symplectic structure on $\mathcal{P}$ constructed by Mukai in [14]. Also, in this case there is a natural algebraic 1 -form on $\mathcal{P}$ such that the symplectic form is the exterior derivative of it.

In this continuation of the papers [4,5] of the second author, our aim is to prove the following (see Theorem 4.6):

Theorem 1.1 The isomorphism $\Phi$ in (1.1) takes the Poisson structure on $\mathcal{M}$ to the Poisson structure on $\mathcal{P}$.

When $N=K_{X}$, related results can be found in [2,7,10]; there the symplectic form on a moduli space of Higgs bundles is compared with the symplectic form on the Hilbert scheme of zero dimensional subschemes of fixed length of the total space of $K_{X}$ (Mukai had shown that this Hilbert scheme has a symplectic structure). 


\section{Hitchin pairs and spectral data}

Let $X$ be an irreducible smooth complex projective curve of genus $g$, and let $N$ be a fixed algebraic line bundle on $X$. A Hitchin pair $(E, \theta)$ is an algebraic vector bundle $E$ on $X$ together with a morphism $\theta: E \longrightarrow E \otimes N$ of $\mathcal{O}_{X}$-modules $[8,15]$.

A Hitchin pair $(E, \theta)$ is stable (respectively, semistable) if

$$
\frac{\operatorname{deg} F}{\operatorname{rk} F}<\frac{\operatorname{deg} E}{\operatorname{rk} E} \quad \text { (respectively, } \frac{\operatorname{deg} F}{\operatorname{rk} F} \leq \frac{\operatorname{deg} E}{\operatorname{rk} E} \text { ) }
$$

for all subbundles $0 \neq F \subsetneq E$ for which $\theta(F) \subset F \otimes N$.

Let $\overline{\mathcal{M}}$ denote the moduli space of all S-equivalence classes of semistable Hitchin pairs (constructed in [15]; see [15, Definition 4.2] for definition of S-equivalence) of fixed rank $r$ and degree $\delta$. Let

$$
\mathcal{M} \subset \overline{\mathcal{M}}
$$

be the open subset of stable Hitchin pairs. We remark that the S-equivalence class of a stable Hitchin pair is the same thing as its isomorphism class, so this moduli space parametrizes isomorphism classes of objects.

Lemma 2.1 The moduli space $\mathcal{M}$ of stable Hitchin pairs $(E, \theta: E \rightarrow E \otimes N)$, with $\operatorname{deg}(E)=\delta, \operatorname{rk}(E)=r$, and $N=K_{X}(D)$ for an effective divisor $D \geq 0$, is smooth.

Proof This is well-known, but since we did not find a reference, we include a sketch of the proof. Fix a line bundle $\Delta \in \operatorname{Pic}^{\delta}(X)$ on $X$ of degree $\delta$, and let $\mathcal{M}_{\Delta}$ be the moduli space of stable Hitchin pairs with fixed determinant $\operatorname{det}(E) \cong \Delta$ and $\operatorname{tr}(\theta)=0$. Consider the determinant morphism

$$
\text { det }: \mathcal{M} \longrightarrow \operatorname{Pic}^{\delta}(X) \times H^{0}(N)
$$

sending $(E, \theta)$ to $(\operatorname{det}(E), \operatorname{tr}(\theta))$. This morphism is surjective, and the target is smooth, so to prove smoothness of $\mathcal{M}$ it is enough to show that the morphism is flat and the fibers are smooth. Note that the fiber over a point $(L, \alpha)$ is isomorphic to the moduli space $\mathcal{M}_{\Delta}$. Indeed, let $\eta \in J(X)$ such that $\eta^{r}=L \otimes \Delta^{-1}$, and send $(E, \theta) \in \mathcal{M}_{\Delta}$ to $\left(E \otimes \eta, \theta+\frac{1}{r} \alpha\right)$. We can choose a root $\eta$ in an étale neighbourhood of $\Delta$, so the determinant morphism is étale locally trivial, and in particular it is flat. Therefore, it only remains to prove that $\mathcal{M}_{\Delta}$ is smooth.

The deformation theory of the moduli space $\mathcal{M}$ of Hitchin pairs of fixed degree is governed by the complex (in degrees 0 and 1)

$$
C_{\mathcal{E}_{n d}}: \mathcal{E} n d(E) \stackrel{[\cdot, \theta]}{\longrightarrow} \mathcal{E} n d(E) \otimes N
$$

This is proved in [3]. In particular, the infinitesimal deformations are given by $\mathbb{H}^{1}\left(C_{\mathcal{E} n d}^{\bullet}\right)$, and $\mathbb{H}^{2}\left(C_{\mathcal{E}}^{\bullet}{ }_{n d}\right)$ is the obstruction space. Furthermore, the exact sequence of complexes

$$
0 \longrightarrow \mathcal{E} n d(E) \otimes N[-1] \longrightarrow C_{\mathcal{E}}^{\bullet}{ }_{n d} \longrightarrow \mathcal{E} n d(E) \longrightarrow 0
$$


$([-1]$ means that the sheaf is placed in degree 1$)$ gives an exact sequence

$$
0 \longrightarrow \mathbb{H}^{0}\left(C_{\mathcal{E}}^{\bullet}\right) \longrightarrow H^{0}(\mathcal{E} n d(E)) \stackrel{[\cdot, \theta]}{\longrightarrow} H^{0}(\mathcal{E} n d(E) \otimes N)
$$

and then $\mathbb{H}^{0}\left(C_{\mathcal{E}_{n d}}^{\bullet}\right)$ represents the endomorphisms of $(E, \theta)$ in the category of Hitchin pairs, i.e., endomorphisms of $E$ which commute with $\theta$.

On the other hand, the deformation theory of the moduli space $\mathcal{M}_{\Delta}$ of stable Hitchin pairs of fixed determinant is given by the complex

$$
C_{a d}^{\bullet}: \operatorname{ad}(E) \stackrel{[\cdot, \theta]}{\longrightarrow} \operatorname{ad}(E) \otimes N
$$

This can be checked by a straightforward modification of the proofs in [3], requiring at each step that the deformation of $E$ does not change its determinant, and that the deformation of $\theta$ preserves the vanishing of its trace.

It is well-known that if $E$ is a stable vector bundle, then its only endomorphisms are given by scalar multiplication (cf. [11, Proposition 1.2.7 and Corollary 1.2.8]). A straightforward modification of the usual argument for vector bundles shows that this is also true in the category of Hitchin pairs, i.e., if $(E, \theta)$ is stable as a Hitchin pair, and $\varphi: E \rightarrow E$ is a homomorphism which commutes with $\theta$, then $\varphi$ is the multiplication by a scalar. Therefore, using (2.2) we have

$$
\mathbb{H}^{0}\left(C_{\mathcal{E} n d}^{\bullet}\right)=\mathbb{C} \cdot \mathrm{id}_{E}
$$

Using the exact sequence

$$
0 \longrightarrow \mathbb{H}^{0}\left(C_{a d}^{\bullet}\right) \longrightarrow H^{0}(\operatorname{ad}(E)) \stackrel{[\cdot, \theta]}{\longrightarrow} H^{0}(\operatorname{ad}(E) \otimes N)
$$

we obtain

$$
\mathbb{H}^{0}\left(C_{a d}^{\bullet}\right)=0
$$

because this cohomology is given by the endomorphisms of $E$ which are traceless and commute with $\theta$.

We claim that $\mathbb{H}^{2}\left(C_{a d}^{\bullet}\right)$ vanishes. Indeed, this group is dual to

$$
\mathbb{H}^{0}\left(\operatorname{ad}(E) \otimes N^{-1} \otimes K_{X} \longrightarrow \operatorname{ad}(E) \otimes K_{X}\right)
$$

If $N \cong K_{X}$, then this is just $\mathbb{H}^{0}\left(C_{a d}^{\bullet}\right)$, which we have just seen that it is zero (2.3). On the other hand, if $N \cong K_{X}(D)$ with $D>0$ an effective, nonzero divisor, then note that the complex which appears in (2.4) is just $C_{a d}^{\bullet} \otimes \mathcal{O}_{X}(-D)$. We have an exact sequence of complexes

$$
0 \longrightarrow C_{a d}^{\bullet} \otimes \mathcal{O}_{X}(-D) \longrightarrow C_{a d}^{\bullet} \longrightarrow C_{a d}^{\bullet} \otimes \mathcal{O}_{D} \longrightarrow 0
$$


The associated long exact cohomology sequence starts as

$$
0 \longrightarrow \mathbb{H}^{0}\left(C_{a d}^{\bullet} \otimes \mathcal{O}_{X}(-D)\right) \longrightarrow \mathbb{H}^{0}\left(C_{a d}^{\bullet}\right) \longrightarrow \mathbb{H}^{0}\left(\left.C_{a d}^{\bullet}\right|_{D}\right)
$$

The vanishing of $\mathbb{H}^{0}\left(C_{a d}^{\bullet}\right)$ (2.3) implies the vanishing of $\mathbb{H}^{0}\left(C_{a d}^{\bullet} \otimes \mathcal{O}_{X}(-D)\right)$. So we have proved that the obstruction space $\mathbb{H}^{2}\left(C_{a d}^{\bullet}\right)$ vanishes.

Summing up, there are no infinitesimal isomorphisms, and the obstruction space vanishes, so $\mathcal{M}_{\Delta}$ is smooth, and then the smoothness of $\operatorname{Pic}^{\delta}(X)$ implies the smoothness of $\mathcal{M}$.

In the sequel we assume that one of the following four assumptions hold:

(1) $g \geq 2$;

(2) $g=1, N=K_{X}$ and $\operatorname{gcd}(r, \delta)=1$;

(3) $g=1$ and $\operatorname{deg}\left(N \otimes K_{X}^{-1}\right)>0$;

(4) $g=0$ and $\operatorname{deg}\left(N \otimes K_{X}^{-1}\right) \geq 3$.

This assumption implies the existence of stable Hitchin pairs [13, Remark 3.4], and hence $\mathcal{M}$ is nonempty.

We will now recall the spectral construction, which is a bijective correspondence between Hitchin pairs $(E, \theta)$ and certain sheaves $\mathcal{L}$ on the total space

$$
p: S:=\mathbb{V}(N) \longrightarrow X
$$

of the line bundle $N$. This construction is in $[9, \S 5]$ for smooth spectral curves, $[1$, p. 173-174, Proposition 3.6] for integral spectral curves, and [17, p. 18, Lemma 6.8] in general (see also [1, p. 173-174, Remark 3.7] and [16]).

Consider the projection $p$ in (2.5). Let $x$ denote the tautological section of $p^{*} N$ on $S=\mathbb{V}(N)$. For a Hitchin pair $(E, E \stackrel{\theta}{\longrightarrow} E \otimes N)$, we define the associated sheaf $\mathcal{L}$ using the following short exact sequence of coherent sheaves on $S$

$$
0 \longrightarrow p^{*}\left(E \otimes N^{\vee}\right) \stackrel{h}{\longrightarrow} p^{*} E \longrightarrow \mathcal{L} \longrightarrow 0
$$

where $p$ is the projection in (2.5) and $h:=p^{*} \theta-x$, with $x$ being the tensor product with the above mentioned tautological section $x$; the homomorphism $E \otimes N^{\vee} \longrightarrow E$ given by $\theta$ is denoted here by $\theta$ also. Throughout, the dual will be denoted by the superscript " $\vee$ ". The homomorphism $h$ in (2.6) is injective (it is an isomorphism over the generic point of $S$ ), so (2.6) is indeed a short exact sequence of coherent sheaves. The spectral curve

$$
Y \subset S
$$

for $(E, \theta)$ is the subscheme defined by the characteristic polynomial

$$
\operatorname{det}\left(p^{*} \theta-x\right)=0 .
$$


Equivalently, the spectral curve $Y$ is the subscheme defined by the 0 -th Fitting ideal sheaf

$$
\operatorname{Fitt}_{0}(\mathcal{L}) \subset \mathcal{O}_{S}
$$

To see this equivalence, note that (2.6) gives a presentation of $\mathcal{L}$, and, by definition, the 0 -th Fitting ideal is the ideal generated by the codimension zero minors of the morphism $h$ in (2.6). Since the ranks of the source and target of $h$ are equal, we only have one minor which is the determinant $\operatorname{det}(h)=\operatorname{det}\left(p^{*} \theta-x\right)$, in other words the characteristic polynomial of $\theta$. We have the following diagram

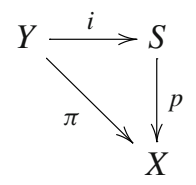

The spectral curve $Y$ is given as the zero of $\operatorname{det}(h)$, which is a section of $p^{*} N^{r}$, and it is proper over $X$, of degree $r$. Note that $\mathcal{L}$ is pure of dimension 1 because $p_{*} \mathcal{L}=E$ is torsion free. The Euler characteristic of $\mathcal{L}$ is $\chi(\mathcal{L})=\chi(E)=\operatorname{deg}(E)+r(1-g)$. We collect all these properties in the following definition.

Definition 2.2 A spectral sheaf $\mathcal{L}$ on $S$, with invariants $r$ and $\delta$, is a coherent sheaf such that:

(1) $\mathcal{L}$ is pure of dimension 1.

(2) The subscheme $Y \subset S$ defined by its 0-th Fitting ideal is in the divisor class given by $p^{*} N^{r}$.

(3) The projection $Y \longrightarrow X$ is proper, finite of degree $r$.

(4) $\chi(\mathcal{L})=\delta+r(1-g)$.

If the spectral curve $Y \subset S$ associated to a spectral sheaf $\mathcal{L}$ is smooth, then there is a line bundle $L$ on $Y$ such that $\mathcal{L}=i_{*} L$, where $i$ is the map in (2.7) ( [1, p. 173-174, Remarks 3.7 and 3.8]).

Conversely, let $\mathcal{L}$ be a spectral sheaf on $S$. The push-forward

$$
E=p_{*} \mathcal{L}
$$

is then a torsion free coherent sheaf, of rank $r$ and degree $\delta$. Define the Hitchin pair ( $E, \theta)$ by setting $\theta=p_{*} x$, where $p$ is the projection in (2.7). More explicitly, consider the multiplication by the tautological section $x$ of $p^{*} N$

$$
\mathcal{L} \stackrel{x}{\longrightarrow} \mathcal{L} \otimes p^{*} N
$$

and then $\theta=p_{*} x$ is defined as the push-forward of this homomorphism, using the projection formula

$$
\theta: E=p_{*} \mathcal{L} \longrightarrow p_{*}\left(\mathcal{L} \otimes p^{*} N\right)=\left(p_{*} \mathcal{L}\right) \otimes N=E \otimes N
$$


[see (2.8)]. The above reversible construction gives a correspondence between the Hitchin pairs $(E, \theta)$ and the spectral sheaves $\mathcal{L}$ on $S$

Note that the push-forward $p_{*} \mathcal{L}$ is coherent if and only if the projection $Y \longrightarrow X$ is proper, and this holds if and only if the closure of $Y$ in $\bar{S}=\mathbb{P}\left(N \oplus \mathcal{O}_{X}\right)$ lies in $S$ ( [17, p. 18, Lemma 6.8]).

We shall now recall the definition of stability given by Simpson ([17]) for a spectral sheaf $\mathcal{L}$. Let

$$
P(\mathcal{L}, m)=\chi\left(\mathcal{L} \otimes p^{*} \mathcal{O}_{X}(m)\right)
$$

be its Hilbert polynomial. Its degree $d$ is equal to the dimension of the support of $\mathcal{L}$, and the leading coefficient is $a m^{d} / d$ !, where $a=a(\mathcal{L})$ is an integer which we call the rank of $\mathcal{L}$. We say that $\mathcal{L}$ is stable (respectively, semistable) if for all proper subsheaves $0 \neq \mathcal{L}^{\prime} \subset \mathcal{L}$,

$a(\mathcal{L}) P\left(\mathcal{L}^{\prime}, m\right)<a\left(\mathcal{L}^{\prime}\right) P(\mathcal{L}, m) \quad\left(\right.$ respectively, $\left.a(\mathcal{L}) P\left(\mathcal{L}^{\prime}, m\right) \leq a\left(\mathcal{L}^{\prime}\right) P(\mathcal{L}, m)\right)$

It is easy to see that if $\mathcal{L}$ is semistable, then it has pure dimension, i.e., the dimension of the support of every nonzero subsheaf is the same as the dimension of the support of $\mathcal{L}$. Note that, in the spectral construction, the condition that $\mathcal{L}^{\prime}$ is a subsheaf of $\mathcal{L}$ translates into the condition that the subbundle

$$
E^{\prime}:=p_{*} \mathcal{L}^{\prime} \subset E:=p_{*} \mathcal{L}
$$

satisfies the condition $\theta\left(E^{\prime}\right) \subset E^{\prime} \otimes N$. It follows that a Hitchin pair is stable (respectively, semistable) if and only if the corresponding spectral sheaf on $S$ is stable (respectively, semistable) [17, p. 19, Corollary 6.9].

The spectral construction can be carried out for families, so we get an isomorphism between the moduli functors. Since the stability conditions coincide, we get an isomorphism between the moduli space of stable Hitchin pairs $\mathcal{M}$ or rank $r$ and degree $\delta$ and the moduli space $\mathcal{P}$ of stable spectral sheaves with invariants $r$ and $\delta$

$$
\Phi: \mathcal{M} \longrightarrow \mathcal{P}
$$

In particular, both moduli spaces, $\mathcal{M}$ and $\mathcal{P}$, are smooth (Lemma 2.1).

We are interested in calculating the differential of the isomorphism $\Phi$ in (2.9). More generally, we will give an isomorphism between the infinitesimal deformation space of a Hitchin pair $(E, \theta)$ and the infinitesimal deformation space of the corresponding sheaf $\mathcal{L}$ on $S$.

\section{Poisson structure on the moduli spaces}

The infinitesimal deformation space of a spectral sheaf (Definition 2.2) $\mathcal{L}$ is given by

$$
\operatorname{Ext}^{1}(\mathcal{L}, \mathcal{L})
$$


[5, p. 425, (3.1)]. To calculate the dual of this vector space we will use Serre duality. Recall that $\mathcal{L}$ is a coherent sheaf on the surface $S=\mathbb{V}(N)$ in (2.5) which is not projective. However, it has a projective compactification

$$
j: S \longrightarrow \bar{S}=\mathbb{P}\left(N \oplus \mathcal{O}_{X}\right)
$$

furthermore, the direct image $j_{*} \mathcal{L}$ is a coherent sheaf on $\bar{S}$, because the closure, in $\bar{S}$, of the support of $\mathcal{L}$ does not meet the boundary $\bar{S} \backslash S$ (this argument is in [17, p. 18, Lemma 6.8]). We have

$$
\begin{aligned}
\operatorname{Ext}^{1}(\mathcal{L}, \mathcal{L})^{\vee} & =\operatorname{Ext}_{\mathcal{O}_{\bar{S}}}^{1}\left(j_{*} \mathcal{L}, j_{*} \mathcal{L}\right)^{\vee} \cong \operatorname{Ext}_{\mathcal{O}_{\bar{S}}}^{1}\left(j_{*} \mathcal{L}, j_{*} \mathcal{L} \otimes K_{\bar{S}}\right) \\
& =\operatorname{Ext}^{1}\left(\mathcal{L}, \mathcal{L} \otimes K_{S}\right)
\end{aligned}
$$

where the second isomorphism is Serre duality on the projective surface $\bar{S}$.

Let $\mathcal{P}$ be the moduli space of stable spectral sheaves on $S$ (cf. (2.9)). The tangent space $T_{[\mathcal{L}]} \mathcal{P}$ at the point corresponding to $\mathcal{L}$ is canonically identified with $\operatorname{Ext}^{1}(\mathcal{L}, \mathcal{L})$, and the cotangent space $T_{[\mathcal{L}]}^{\vee} \mathcal{P}$ is canonically identified with $\operatorname{Ext}^{1}\left(\mathcal{L}, \mathcal{L} \otimes K_{S}\right)$ (see (3.2)).

Henceforth assume that the line bundle $N \otimes K_{X}^{-1}$ on $X$ admits a nonzero section. We fix a nonzero section

$$
\sigma_{0} \in H^{0}\left(X, N \otimes K_{X}^{-1}\right) \backslash\{0\}
$$

Since the anticanonical line bundle $K_{S}^{\vee}$ of the surface $S$ in (2.5) is identified with $p^{*}\left(N \otimes K_{X}^{-1}\right)$, where $p$ is the projection in (2.5), we have

$$
s:=p^{*} \sigma_{0} \in H^{0}\left(S, K_{S}^{\vee}\right)
$$

where $\sigma_{0}$ is the section in (3.3). This $s$ produces an algebraic Poisson structure on $\mathcal{P}$

$$
B: T^{\vee} \mathcal{P} \longrightarrow T \mathcal{P}
$$

[5,18]. We will recall below the fiberwise construction of $B$.

Tensoring with the section $s$ produces a homomorphism $\mathcal{L} \otimes K_{S} \longrightarrow \mathcal{L}$. Consequently, we obtain a homomorphism

$$
T_{[\mathcal{L}]}^{\vee} \mathcal{P} \cong \operatorname{Ext}^{1}\left(\mathcal{L}, \mathcal{L} \otimes K_{S}\right) \longrightarrow \operatorname{Ext}^{1}(\mathcal{L}, \mathcal{L}) \cong T_{[\mathcal{L}]} \mathcal{P}
$$

The isomorphism $\operatorname{Ext}^{1}(\mathcal{L}, \mathcal{L}) \cong T_{[\mathcal{L}]} \mathcal{P}$ is a consequence of the fact that the infinitesimal deformations of $\mathcal{L}$ are parametrized by $\operatorname{Ext}^{1}(\mathcal{L}, \mathcal{L})$, while the isomorphism $T_{[\mathcal{L}]}^{\vee} \mathcal{P} \cong \operatorname{Ext}^{1}\left(\mathcal{L}, \mathcal{L} \otimes K_{S}\right)$ follows from (3.2). Let $B([\mathcal{L}])$ be the restriction to the fiber over the point $[\mathcal{L}]$ of the bundle map $B$ in (3.5). This homomorphism $B([\mathcal{L}])$ coincides with the one in (3.6) (see [5, p. 428, Formula (4.4)]). 
On the other hand, the infinitesimal deformation space of a Hitchin pair $(E, \theta)$ is given by the first hypercohomology of a complex

$$
\mathbb{H}^{1}\left(E^{\vee} \otimes E \stackrel{[\cdot, \theta]}{\longrightarrow} E^{\vee} \otimes E \otimes N\right)
$$

(see [4, p. 399, Proposition 3.1.2], [13, p. 271, Proposition 7.1], [3, p. 220, Theorem 2.3]). The dual vector space

$$
\mathbb{H}^{1}\left(E^{\vee} \otimes E \stackrel{[\cdot, \theta]}{\longrightarrow} E^{\vee} \otimes E \otimes N\right)^{\vee}
$$

is calculated using Serre duality for hypercohomologies. Denote by $A^{\bullet}$ the complex

$$
A^{\bullet}: \quad E^{\vee} \otimes E \stackrel{[\cdot, \theta]}{\longrightarrow} E^{\vee} \otimes E \otimes N
$$

concentrated in degrees 0 and 1 . Serre duality gives an isomorphism

$$
\mathbb{H}^{1}\left(A^{\bullet}\right)^{\vee} \stackrel{\cong}{\longrightarrow} \mathbb{H}^{-1}\left(A^{\bullet \vee}[1] \otimes K_{X}\right)=\mathbb{H}^{1}\left(A^{\bullet \vee}[-1] \otimes K_{X}\right)
$$

[12, p. 67, Theorem 3.12]. The dual complex

$$
A^{\bullet \vee}: \quad E \otimes E^{\vee} \otimes N^{\vee} \stackrel{[\cdot, \theta]^{t}}{\longrightarrow} E \otimes E^{\vee}
$$

is concentrated in degrees -1 and 0 . We identify $E \otimes E^{\vee}$ and $E^{\vee} \otimes E$ by switching the factors; then the morphism $[\cdot, \theta]^{t}$ becomes $[\theta, \cdot]$, so

$$
A^{\bullet \vee}: \quad E^{\vee} \otimes E \otimes N^{\vee} \stackrel{[\theta, \cdot]}{\longrightarrow} E^{\vee} \otimes E
$$

Finally we shift the complex in (3.8) by [-1]. Recall that, when we shift a complex by one unit, we multiply by -1 all the differentials [12, p. 28, Definition 2.4], so we get the complex, concentrated in degrees 0 and 1

$$
A^{\bullet \vee}[-1] \otimes K_{X}: \quad E^{\vee} \otimes E \otimes N^{\vee} \otimes K_{X} \stackrel{[\cdot, \theta]}{\longrightarrow} E^{\vee} \otimes E \otimes K_{X}
$$

Remark 3.1 In [4, p. 402, Proposition 3.1.10] the morphism is actually [ $\theta$, .] instead of the homomorphism $[\cdot, \theta]$ in (3.7). Of course there is an isomorphism between the two complexes (multiplication by -1 in one term and +1 in the other).

As in (2.9), let $\mathcal{M}$ be a moduli space of stable Hitchin pairs such that the rank and degree of the vector bundle underlying a Hitchin pair are $r$ and $\delta$ respectively. Using the section $\sigma_{0}$ in (3.3) an algebraic Poisson structure

$$
B^{H}: T^{\vee} \mathcal{M} \longrightarrow T \mathcal{M}
$$


on $\mathcal{M}$ is constructed [4, $\S 4.3]$, [13, p. 278, Corollary 7.15]. We recall below a fiberwise construction of the homomorphism $B^{H}$ in (3.10).

Tensoring with the section $\sigma_{0}$ in (3.3) induces homomorphisms $N^{\vee} \otimes K_{X} \longrightarrow \mathcal{O}_{X}$ and $K_{X} \longrightarrow N$. Using these we obtain a homomorphism of complexes

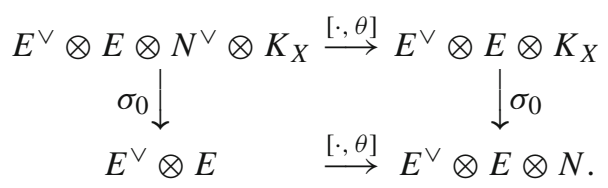

The morphism of complexes in (3.11) gives a morphism of cohomologies

$$
\begin{gathered}
\mathbb{H}^{1}\left(E^{\vee} \otimes E \otimes N^{\vee} \otimes K_{X} \quad \stackrel{[\cdot, \theta]}{\longrightarrow} E^{\vee} \otimes E \otimes K_{X}\right) \\
\longrightarrow \mathbb{H}^{1}\left(E^{\vee} \otimes E \quad \stackrel{[\cdot, \theta]}{\longrightarrow} E^{\vee} \otimes E \otimes N\right) .
\end{gathered}
$$

We have $T_{[(E, \theta)]} \mathcal{M}=\mathbb{H}^{1}\left(E^{\vee} \otimes E \stackrel{[\cdot, \theta]}{\longrightarrow} E^{\vee} \otimes E \otimes N\right)$, because the infinitesimal deformations of $(E, \theta)$ are parametrized by $\mathbb{H}^{1}\left(E^{\vee} \otimes E \stackrel{[\cdot, \theta]}{\longrightarrow} E^{\vee} \otimes E \otimes N\right)$ (see (3.7)); this and (3.9) together imply that

$$
T_{[(E, \theta)]}^{\vee} \mathcal{M}=\mathbb{H}^{1}\left(E^{\vee} \otimes E \otimes N^{\vee} \otimes K_{X} \stackrel{[\cdot, \theta]}{\longrightarrow} E^{\vee} \otimes E \otimes K_{X}\right)
$$

Using (3.13) and (3.7), the homomorphism in (3.12) becomes a homomorphism

$$
T_{[(E, \theta)]}^{\vee} \mathcal{M} \longrightarrow T_{[(E, \theta)]} \mathcal{M}
$$

This homomorphism coincides with $B^{H}([(E, \theta)])$ in $(3.10)[4, \S 4.3]$, [13, p. 278, Corollary 7.15].

Remark 3.2 If we compare (3.11) with the commutative diagram in [4, Remark 1.3.3], we see that the sign is changed in the vertical left arrow and in one of the horizontal arrows (there is $[\theta, \cdot]$ in $[4, \operatorname{Remark} 1.3 .3]$ as opposed to $[\cdot, \theta]$ here).

\section{Comparison of Poisson structures}

We shall compare the Poisson structures $B$ and $B^{H}$, constructed in (2.9) and (3.10) respectively, using the isomorphism $\Phi$ in (2.9).

We first recall that the global Ext can be calculated using locally free resolutions and hypercohomology. 
Lemma 4.1 ( [6, Corollary 2 to Theorem 4.2.1]). Let $V$ and $W$ be coherent sheaves on a scheme T. Let

$$
\cdots \longrightarrow L^{-3} \longrightarrow L^{-2} \longrightarrow L^{-1} \longrightarrow L^{0} \longrightarrow V \longrightarrow 0
$$

be a resolution of $V$ by finitely generated locally free sheaves. Then there is an isomorphism

$$
\operatorname{Ext}^{i}(V, W) \cong \mathbb{H}^{i}\left(\mathcal{H} \text { om }\left(L^{\bullet}, W\right)\right)
$$

which is functorial on $W$.

Proposition 4.2 Let $W$ be a coherent sheaf on the surface $S$ in (2.5). Let $x: W \longrightarrow$ $W \otimes p^{*} N$ be multiplication by the tautological section of $p^{*} N$ on $S$, where $p$ is the projection in (2.5). Then for any $(E, \theta) \in \mathcal{M}$ [see (2.9)] there is an isomorphism

$$
\varphi_{W}: \operatorname{Ext}^{1}(\mathcal{L}, W) \stackrel{\cong}{\longrightarrow} \mathbb{H}^{1}\left(E^{\vee} \otimes p_{*} W \stackrel{f_{W}}{\longrightarrow} E^{\vee} \otimes N \otimes p_{*} W\right),
$$

where $\mathcal{L}=\Phi((E, \theta))$ [see (2.9)], $f_{W}=\theta^{\vee} \otimes 1-1 \otimes p_{*} x$, and $f_{W}$ is seen as a complex concentrated in degrees 0 and 1 . This isomorphism is functorial on $W$, meaning a homomorphism $V \longrightarrow W$ of coherent sheaves on $S$ induces a commutative diagram

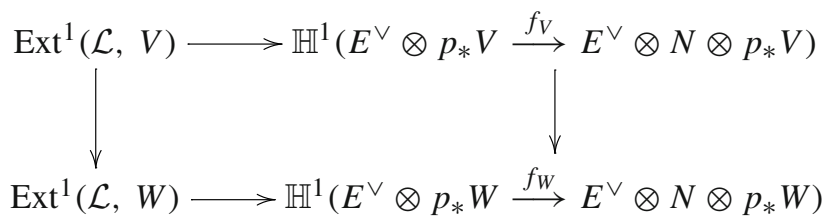

Proof The short exact sequence in (2.6) gives a locally free resolution $L^{\bullet}$ of $\mathcal{L}$ (concentrated on degrees -1 and 0 )

$$
L^{\bullet}: \quad p^{*}\left(E \otimes N^{\vee}\right) \stackrel{h}{\longrightarrow} p^{*} E .
$$

For any sheaf $W$ on $S$, Lemma 4.1 gives an isomorphism functorial for $W$

$$
\operatorname{Ext}^{1}(\mathcal{L}, W)=\mathbb{H}^{1}\left(\mathcal{H o m}\left(L^{\bullet}, W\right)\right)
$$

Consider the diagram

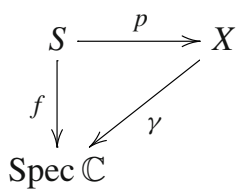


where $f$ and $\gamma$ are the structure morphisms. Note that the hypercohomology of any complex $D^{\bullet}$ on $S$ can be calculated as the cohomology of the complex $R f_{*} D^{\bullet}$ (where $R f_{*}$ is the derived functor between the derived categories). We have

$$
R f_{*}=R\left(\gamma_{*} \circ p_{*}\right) \stackrel{\cong}{\longrightarrow} R \gamma_{*} \circ R p_{*} .
$$

The morphism $p$ is affine, hence $p_{*}$ is exact and then $R p_{*}=p_{*}$. If we apply this observation to the complex $\mathcal{H o m}\left(L^{\bullet}, W\right)$, we get

$$
\mathbb{H}^{1}\left(\mathcal{H o m}\left(L^{\bullet}, W\right)\right) \stackrel{\cong}{\longrightarrow} \mathbb{H}^{1}\left(p_{*} \mathcal{H o m}\left(L^{\bullet}, W\right)\right)
$$

In view of (4.1) and (4.2), to finish the proof, it only remains to show that the complex $p_{*} \mathcal{H} \operatorname{Hom}\left(L^{\bullet}, W\right)$ is equal to the complex $f_{W}$ in the statement of the proposition.

Applying $\mathcal{H o m}(\cdot, W)$ to the complex $L^{\bullet}$ we obtain a complex concentrated in degrees 0 and 1

$$
\mathcal{H} \text { om }\left(L^{\bullet}, W\right): \quad p^{*} E^{\vee} \otimes W \stackrel{h^{\prime}}{\longrightarrow} p^{*} E^{\vee} \otimes p^{*} N \otimes W,
$$

where $h^{\prime}:=p^{*} \theta^{\vee} \otimes 1-1 \otimes x$. By the projection formula, $p_{*} \mathcal{H} \operatorname{Hom}\left(L^{\bullet}, W\right)$ is $E^{\vee} \otimes p_{*} W=p_{*}\left(p^{*} E^{\vee} \otimes W\right) \stackrel{p_{*} h^{\prime}}{\longrightarrow} p_{*}\left(p^{*} E^{\vee} \otimes p^{*} N \otimes W\right)=E^{\vee} \otimes N \otimes p_{*} W$ where $p_{*} h^{\prime}=\theta^{\vee} \otimes 1-1 \otimes p_{*} x$, and the proposition follows.

Remark 4.3 In the proof of Proposition 4.2, the main step is the isomorphism in (4.2), because it facilitates the passage of objects defined on $S$ to objects defined on $X$. Note that this isomorphism is induced by the push-forward $p_{*}$ using $p$.

Let

$$
d \Phi: T \mathcal{M} \longrightarrow \Phi^{*} T \mathcal{P}
$$

be the differential of the isomorphism $\Phi$ in (2.9).

Lemma 4.4 Let

$$
\varphi: \operatorname{Ext}^{1}(\mathcal{L}, \mathcal{L}) \stackrel{\cong}{\longrightarrow} \mathbb{H}^{1}\left(E^{\vee} \otimes E \stackrel{f_{\mathcal{L}}}{\longrightarrow} E^{\vee} \otimes N \otimes E\right)
$$

be the homomorphism given by Proposition 4.2 for $W=\mathcal{L}$ [see (2.8)]. Then the following diagram is commutative

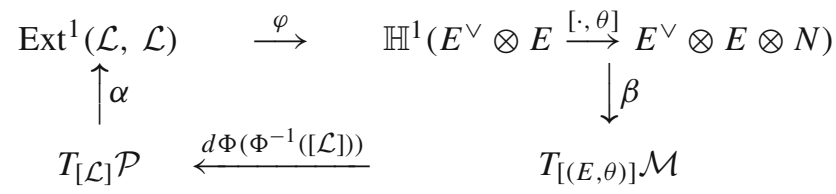


where $\alpha$ and $\beta$ are the infinitesimal deformation maps in (3.1) and (3.7) respectively, and $d \Phi\left(\Phi^{-1}([\mathcal{L}])\right)$ is the homomorphism in $(4.4)$ at $\Phi^{-1}([\mathcal{L}]) \in \mathcal{M}$.

Proof Consider an infinitesimal deformation $\mathcal{L}_{\epsilon}$ of $\mathcal{L}$, i.e., a sheaf on $S \times \operatorname{Spec}(\mathbb{C}[\epsilon] /$ $\left.\left(\epsilon^{2}\right)\right)$ flat over $\operatorname{Spec}\left(\mathbb{C}[\epsilon] /\left(\epsilon^{2}\right)\right)$, that fits in a short exact sequence

$$
0 \longrightarrow \mathcal{L} \stackrel{\epsilon}{\longrightarrow} \mathcal{L}_{\epsilon} \stackrel{\bmod \epsilon}{\longrightarrow} \mathcal{L} \longrightarrow 0
$$

By applying the functor $\operatorname{Hom}(\mathcal{L}, \cdot)$ to this short exact sequence we obtain a long exact sequence

$$
\cdots \longrightarrow \operatorname{Hom}\left(\mathcal{L}, \mathcal{L}_{\epsilon}\right) \longrightarrow \operatorname{Hom}(\mathcal{L}, \mathcal{L}) \stackrel{\delta}{\longrightarrow} \operatorname{Ext}^{1}(\mathcal{L}, \mathcal{L}) \longrightarrow \cdots
$$

the element of $\operatorname{Ext}^{1}(\mathcal{L}, \mathcal{L})$ that corresponds to the infinitesimal deformation $\mathcal{L}_{\epsilon}$ is $\delta$ (id), the image of the identity id $\in \operatorname{Hom}(\mathcal{L}, \mathcal{L})$ by the connecting homomorphism $\delta$.

If we set $W=\mathcal{L}$ in Proposition 4.2, we have $p_{*} W=E$ and $p_{*} x=\theta$; then $f_{W}=[\cdot, \theta]$, and Proposition 4.2 produces the isomorphism $\varphi$.

The element

$$
\beta \circ \varphi \circ \alpha\left(\mathcal{L}_{\epsilon}\right) \in T_{[(E, \theta)]} \mathcal{M}
$$

where $\mathcal{L}_{\epsilon} \in T_{[\mathcal{L}]} \mathcal{P}$ is the element in (4.5), has the following description.

Consider the short exact sequence (4.5). Applying $p_{*}$ to it, we obtain an exact sequence of complexes

$$
0 \longrightarrow K^{\bullet} \stackrel{\epsilon}{\longrightarrow} K_{\epsilon}^{\bullet} \stackrel{\bmod \epsilon}{\longrightarrow} K^{\bullet} \longrightarrow 0
$$

where $K^{\bullet}$ denotes the complex $0 \longrightarrow E \stackrel{\theta}{\longrightarrow} E \otimes N \longrightarrow 0$. Since $K_{\epsilon}^{\bullet}$ in (4.7) is a family of Hitchin pairs on $X$ parametrized by $\operatorname{Spec}\left(\mathbb{C}[\epsilon] /\left(\epsilon^{2}\right)\right)$, it corresponds to an element of $T_{[(E, \theta)]} \mathcal{M}$. This element of $T_{[(E, \theta)]} \mathcal{M}$ coincides with the one in (4.6). Now from the construction of the map $\Phi$ it follows that

$$
d \Phi\left(\Phi^{-1}([\mathcal{L}])\right)\left(\beta \circ \varphi \circ \alpha\left(\mathcal{L}_{\epsilon}\right)\right) \in T_{[\mathcal{L}]} \mathcal{P}
$$

coincides with $\mathcal{L}_{\epsilon} \in T_{[\mathcal{L}]} \mathcal{P}$.

We remark that the key to the commutativity of the diagram in the statement of Lemma 4.4 is that both $\varphi$ and $\Phi^{-1}$ are induced by the push-forward $p_{*}$.

Lemma 4.5 Consider

$$
\begin{aligned}
\varphi_{1} & :=\left(\varphi_{\mathcal{L} \otimes K_{S}}\right)^{-1}: \mathbb{H}^{1}\left(E^{\vee} \otimes E \otimes K_{X} \otimes N^{\vee} \stackrel{[\cdot, \theta]}{\longrightarrow} E^{\vee} \otimes E \otimes K_{X}\right) \\
& \longrightarrow \operatorname{Ext}^{1}\left(\mathcal{L}, \mathcal{L} \otimes K_{S}\right),
\end{aligned}
$$


where $\varphi_{\mathcal{L} \otimes K_{S}}$ is the isomorphism in Proposition 4.2 for $W=\mathcal{L} \otimes K_{S}$. Let $(d \Phi)^{\vee}([\mathcal{L}])$ be dual of the differential of the isomorphism $\Phi$ at $[\mathcal{L}]$. Then the following diagram is commutative

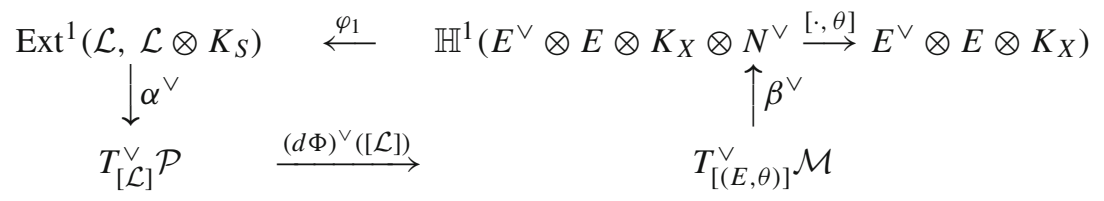

where $\alpha^{\vee}$ and $\beta^{\vee}$ are the natural isomorphisms obtained from (3.2) and (3.9) respectively, and $(d \Phi)^{\vee}$ is the dual of the homomorphism in (4.4).

Proof Consider the homomorphism $\varphi$ in Lemma 4.4. For any

$$
\omega \in \mathbb{H}^{1}\left(E^{\vee} \otimes E \otimes K_{X} \otimes N^{\vee} \stackrel{[\cdot, \theta]}{\longrightarrow} E^{\vee} \otimes E \otimes K_{X}\right)
$$

and $v \in \operatorname{Ext}^{1}(\mathcal{L}, \mathcal{L})$, we have

$$
\varphi_{1}(\omega)(v)=\omega(\varphi(v)) \in \mathbb{C}
$$

recall that

$\mathbb{H}^{1}\left(E^{\vee} \otimes E \otimes K_{X} \otimes N^{\vee} \stackrel{[\cdot, \theta]}{\longrightarrow} E^{\vee} \otimes E \otimes K_{X}\right)=\mathbb{H}^{1}\left(E^{\vee} \otimes E \stackrel{[\cdot, \theta]}{\longrightarrow} E^{\vee} \otimes E \otimes N\right)^{\vee}$ [see (3.13) and (3.7)], and

$$
\operatorname{Ext}^{1}\left(\mathcal{L}, \mathcal{L} \otimes K_{S}\right)=\operatorname{Ext}^{1}(\mathcal{L}, \mathcal{L})^{\vee}
$$

[see (3.2)]. From (4.8) it follows immediately that $\varphi_{1}$ coincides with the dual homomorphism $\varphi^{\vee}$.

Therefore, every homomorphism in the diagram in this lemma is the dual of the corresponding homomorphism in the diagram in Lemma 4.4. Hence the lemma follows from Lemma 4.4 .

The homomorphism $B$ in (3.5) gives an algebraic section

$$
B \in H^{0}\left(\mathcal{P}, \bigwedge^{2} T \mathcal{P}\right)
$$

and the homomorphism $B^{H}$ in (3.10) gives an algebraic section

$$
B^{H} \in H^{0}\left(\mathcal{M}, \bigwedge^{2} T \mathcal{M}\right)
$$

Consider the homomorphism $d \Phi$ in (4.4). We note that

$$
\Phi_{*} B^{H}:=\left(\bigwedge^{2} d \Phi\right)\left(B^{H}\right) \in H^{0}\left(\mathcal{P}, \bigwedge^{2} T \mathcal{P}\right)
$$


is a Poisson structure on $\mathcal{P}$.

Theorem 4.6 The isomorphism $\Phi$ in (2.9) satisfies the condition

$$
\Phi_{*} B^{H}=B
$$

where $\Phi_{*} B^{H}$ and $B$ are the sections in (4.10) and (4.9) respectively.

Proof The section $\sigma_{0} \in H^{0}\left(X, N \otimes K_{X}^{\vee}\right)$ gives a section $s$ of $K_{S}^{\vee}=p^{*}\left(N \otimes K_{X}^{\vee}\right)$, and hence it produces a homomorphism $\mathcal{L} \otimes K_{S} \longrightarrow \mathcal{L}$. We apply Proposition 4.2 to this homomorphism and get the following commutative diagram:

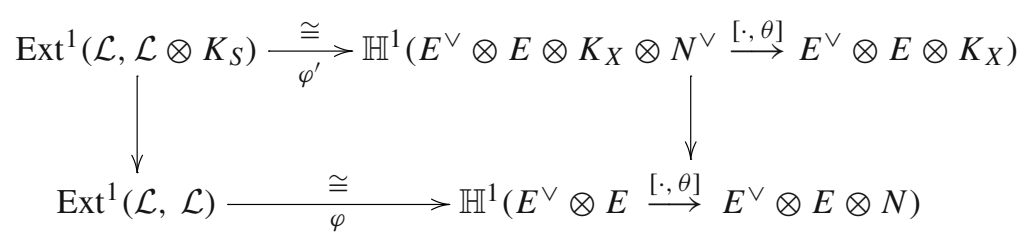

where the left vertical homomorphism is the one in (3.6) and the right vertical homomorphism is the one in (3.12).

Lemma 4.5 shows that the top horizontal homomorphism $\varphi^{\prime}$ in (4.11) coincides with $(d \Phi)^{\vee}([\mathcal{L}])$. We note that $\varphi^{\prime}=\left(\varphi_{1}\right)^{-1}$, where $\varphi_{1}$ is the homomorphism in Lemma 4.5. On the other hand, Lemma 4.4 shows that the bottom horizontal homomorphism in (4.11) coincides with $d \Phi^{-1}([\mathcal{L}])$. The left vertical homomorphism in (4.11) gives $B([\mathcal{L}])$, the Poisson structure on $\mathcal{P}$, while the right vertical homomorphism in (4.11) gives $B^{H}([(E, \theta)])=B^{H}\left(\Phi^{-1}([\mathcal{L}])\right)$, the Poisson structure on $\mathcal{M}$. Consequently, the theorem follows from the commutativity of the diagram in (4.11).

Acknowledgements We thank Oscar García-Prada for helpful discussions on the smoothness and deformation theory of the moduli space of Hitchin pairs. We thank the referee for comments which improved the exposition. The first author is supported by a J. C. Bose fellowship. The third author is supported by Ministerio de Ciencia e Innovación of Spain (grants PID2019-108936GB-C21 and ICMAT Severo Ochoa project CEX2019-000904-S) and CSIC (2019AEP151 and Ayuda extraordinaria a Centros de Excelencia Severo Ochoa 20205CEX001).

Funding Open Access funding provided thanks to the CRUE-CSIC agreement with Springer Nature.; Please verify relation to: Consejo Superior de Investigaciones Cientificas (CSIC);

Open Access This article is licensed under a Creative Commons Attribution 4.0 International License, which permits use, sharing, adaptation, distribution and reproduction in any medium or format, as long as you give appropriate credit to the original author(s) and the source, provide a link to the Creative Commons licence, and indicate if changes were made. The images or other third party material in this article are included in the article's Creative Commons licence, unless indicated otherwise in a credit line to the material. If material is not included in the article's Creative Commons licence and your intended use is not permitted by statutory regulation or exceeds the permitted use, you will need to obtain permission directly from the copyright holder. To view a copy of this licence, visit http://creativecommons.org/licenses/by/4.0/. 


\section{References}

1. Beauville, A., Narasimhan, M.S., Ramanan, S.: Spectral curves and the generalised theta divisor. J. Reine Angew. Math. 398, 169-179 (1989)

2. Biswas, I., Mukherjee, A.: Symplectic structures of moduli space of Higgs bundles over a curve and Hilbert scheme of points on the canonical bundle. Commun. Math. Phys. 221, 293-304 (2001)

3. Biswas, I., Ramanan, S.: An infinitesimal study of the moduli of Hitchin pairs. J. Lond. Math. Soc. 49, 219-231 (1994)

4. Bottacin, F.: Symplectic geometry on moduli spaces of stable pairs. Ann. Sci. École Norm. Sup. 28, 391-433 (1995)

5. Bottacin, F.: Poisson structures on moduli spaces of sheaves over Poisson surfaces. Invent. Math. 121, 421-436 (1995)

6. Grothendieck, A.: Sur quelques points d'algèbre homologique. Tohoku Math. J. 9, 119-221 (1957)

7. Harnad, J., Hurtubise, J.: Multi-Hamiltonian structures for $r$-matrix systems. J. Math. Phys. 49(6), 062903 (2008)

8. Hitchin, N.: The self-duality equations on a Riemann surface. Proc. Lond. Math. Soc. 55, 59-126 (1987)

9. Hitchin, N.: Stable bundles and integrable systems. Duke Math. J. 54, 91-114 (1987)

10. Hurtubise, J.C., Kjiri, M.: Separating coordinates for the generalized Hitchin systems and the classical r-matrices. Commun. Math. Phys. 210, 521-540 (2000)

11. Huybrechts, D., Lehn, M.: The geometry of moduli spaces of sheaves. Aspects of Mathematics, E31, Firedr. Vieweg \& Sohn, Braunschweig (1997)

12. Huybrechts, D.: Fourier-Mukai Transforms in Algebraic Geometry, Oxford Mathematical Monographs. Oxford University Press, Oxford (2006)

13. Markman, E.: Spectral curves and integrable systems. Compos. Math. 93, 255-290 (1994)

14. Mukai, S.: Symplectic structure of the moduli space of sheaves on an abelian or K3 surface. Invent. Math. 77, 101-116 (1984)

15. Nitsure, N.: Moduli space of semistable pairs on a curve. Proc. Lond. Math. Soc. 62, 275-300 (1991)

16. Schaub, D.: Courbes spectrales et compactifications de jacobiennes. Math. Zeit. 227, 295-312 (1998)

17. Simpson, C.: Moduli of representations of the fundamental group of a smooth projective variety. II. Inst. Hautes Études Sci. Publ. Math. 80, 5-79 (1994)

18. Tyurin, A.N.: Symplectic structures on the varieties of moduli of vector bundles on algebraic surfaces with $p_{g}>0$. Math. USSR Izvestiya 33, 139-177 (1989)

Publisher's Note Springer Nature remains neutral with regard to jurisdictional claims in published maps and institutional affiliations. 